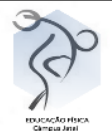

\title{
A educação física adaptada no currículo de formação profissional em educação física
}

\author{
Vivianne Oliveira Gonçalves ${ }^{1}$ \\ Sabrina Toffoli Leite ${ }^{2}$ \\ Edison Duarte ${ }^{3}$
}

\begin{abstract}
Resumo: A partir do Ano Internacional da Pessoa Deficiente (1981) pode-se observar diversas ações que possibilitaram a ampliação do atendimento às pessoas com deficiência, inclusive na área da Educação Física e esporte. Esse artigo discorre sobre a criação de uma disciplina nos cursos de graduação em Educação Física, através da reestruturação curricular de 1990, pontuando questões referentes às ações governamentais e ao percurso para sua inserção no currículo, características da disciplina e estudos realizados acerca do desenvolvimento da mesma no interior dos cursos de formação em Educação Física em diversas regiões do país. Desde então, muitos avanços já foram conseguidos, no entanto, há muito a ser feito, desde a especialização de docentes na área, a organização do currículo de forma adequada (a nomenclatura adequada, assim como os conteúdos a serem abordados), as atividades de extensão, até o avanço nas pesquisas e produção de conhecimento.
\end{abstract}

Palavras - chave: Educação Física Adaptada. Currículo. Formação profissional.

\section{The adapted physical education in the curriculum of courses of physical education}

\begin{abstract}
From the International Year of disabled Person (1981) one can observe several actions that allowed the expansion of service to people with disabilities, including in the area of physical education and sport. This article discusses creating a discipline in undergraduate courses in physical education, through restructuring of 1990 curriculum, punctuating questions relating to governmental actions and the route for its insertion in the curriculum, characteristics of the discipline and studies about the
\end{abstract}

\footnotetext{
${ }^{1}$ Licenciada em Educação Física pela Universidade Federal de Goiás, Mestre em Educação Física pela Universidade Estadual de Campinas e Doutora em Ciências da Atividade Física e do Esporte pela Universidad de Castilla La-Mancha (UCLM-Espanha). Atualmente é docente dos cursos de Educação Física e do Programa de Pós-Graduação em Educação da Universidade Federal de Jataí. Atua nas seguintes áreas: imagem corporal, corpo, gênero e sexualidade, e como pesquisadora nos grupos de Pesquisa Multidisciplinaridade e Saúde, e NESEC - Núcleo de Estudos Sociedade, Educação e Cultura. E-mail: vivianefef@ gmail.com

${ }^{2}$ Possui graduação em Educação Física pela Universidade Estadual de Campinas (UNICAMP), Especialização em Fisiologia do Exercício, Mestrado e Doutorado em Educação Física - Ciência do Desporto pela UNICAMP. Atualmente é Docente Adjunto da Universidade Federal Jataí, no curso de Educação Física. Atua nas seguintes áreas: fisiologia cardiovascular, atividade física e exercício para grupos especiais, qualidade de vida e saúde. Email: sabrina.toffoli@gmail.com

${ }^{3}$ Possui graduação em Fisioterapia pela PUC-Campinas, mestrado pela Universidade Estadual de Campinas e doutorado em Anatomia Humana pela Universidade de São Paulo. Atualmente é professor titular na Faculdade de Educação Física, da Universidade Estadual de Campinas. É membro do Conselho Gestor da Academia Paralímpica Brasileira; Classificador funcional da modalidade Esgrima em Cadeira de Rodas pelo Comitê Paralímpico Brasileiro e pela International Wheelchair \& Amputee Sports Federation. Sócio fundador da Sociedade Brasileira de Atividade Motora Adaptada - SOBAMA. E-mail: edison@ fef.unicamp.br
} 


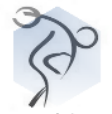

development within the training courses in physical education in various regions of the country. Since then, many advances have been achieved, however, there is much to be done, since the specialization of teachers in the area, the Organization of the curriculum as appropriate (proper nomenclature, as well as the content to be covered), extension activities, until the advance on the research and production of knowledge.

Keywords: Adapted Physical Education. Curriculum. Professional training.

\section{INTRODUÇÃO}

Atualmente, tem-se intensificado o debate acerca do papel dos discursos e práticas curriculares na preparação de professores e de futuras gerações, nos valores de apreciação da diversidade cultural e de desafio a preconceitos ligados ao gênero, raça, religião, deficiências e outros. No Brasil, o campo da educação vem abarcando essas discussões de forma progressiva a partir dos anos 80, especialmente após 1981, Ano Internacional da Pessoa Deficiente, o que contribuiu posteriormente para a inserção de uma disciplina que abordasse a problemática da pessoa com deficiência nos cursos superiores de Educação Física, Arquitetura e outros (CARMO, 1991).

Autores como Araújo (1998) e Silva e Araújo (2005) designaram esse período como institucionalização da Educação Física Adaptada (EFA). No âmbito nacional, o país passava por um período de redemocratização que se tornou propício para a configuração de um amplo movimento de renovação crítica nas distintas práticas escolares, inclusive na área de Educação Física (EF), com consequências nas políticas e práticas de formação e atuação profissional dos professores da área. Já no âmbito internacional, configuraram-se várias ações e movimentos de luta e defesa pela integração e participação social das pessoas com deficiência.

Para Gonçalves (2002), a inserção da disciplina nos currículos faz parte de uma mudança paradigmática na área da EF e crítica à visão funcionalista do movimento humano e à preparação do profissional para atuar com uma população com o estereótipo de corpos fortes e saudáveis. Segundo Betti e Betti (1996), essa discussão ocorre no final da década de 1980, culminando com a proposta de um currículo do tipo técnico-científico em contraposição ao modelo tradicional-desportivo, dominante na área. De acordo com Costa (1992), desde seu início, justificou-se a inclusão da disciplina EFA nos currículos dos Cursos de Educação Física por meio de alguns argumentos, entre eles, o de colocar em discussão a concepção 


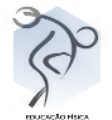

dominante de aptidão física, considerando a pessoa deficiente, que até então não era levada em conta nos cursos de graduação em EF.

Por outro lado, se um dos problemas ocorridos no período da inclusão da EFA nos currículos das Instituições de Ensino Superior (IES) tenha sido a falta de profissional qualificado para ministrar a disciplina; atualmente, a formação do profissional ocorre em vários níveis: Graduação, Especialização, Mestrado e Doutorado (DUARTE, 2004). No entanto, alguns questionamentos continuam em pauta, tais como a nomenclatura adequada ou a população a ser contemplada na disciplina.

Outro ponto importante nesse processo de consolidação da EFA foi a disseminação do conhecimento por profissionais da área. Utilizando como fundamentação alguns desses estudos, o texto aqui apresentado aponta questões referentes à EFA e à inclusão da mesma nos cursos de formação do profissional e professor de EF.

\section{FORMAÇÃO PROFISSIONAL EM EDUCAÇÃO FÍSICA ADAPTADA: principais ações governamentais}

A história da Educação Especial no Brasil foi se modificando substancialmente ao longo dos anos, em termos de ações governamentais. Diversos autores apontaram essas modificações, entre eles, Carmo (1991), Mazzota (1996), Araújo (1998). Nesse sentido e apoiando-se nesses autores, são assinaladas algumas ações e políticas governamentais enfocando principalmente a formação de recursos humanos voltados à prática de atividades física às pessoas com deficiência, ao longo das últimas décadas.

Estudos realizados por Lima (1998) demonstram que o poder público somente mostrou preocupação com a Educação Especial em meados de 1972, através do I Plano Nacional de Desenvolvimento- I PND- no denominado Plano Setorial de Educação e Cultura (PSEC). Neste, são apontadas as diretrizes da Educação Especial, que também se fariam presentes no II PSEC 1975/1979. Entre outras ações, destacam-se: a criação do Centro Nacional de Educação Especial (CENESP) em 1973, e o III Plano Setorial de Educação, Cultura e Desporto (PSECD), nos anos 80. 
Mas, é a partir de 1981, que se intensificam as ações governamentais voltadas ao atendimento da população com deficiência, com as pressões realizadas pela Organização das Nações Unidas (ONU) que, através da proclamação da Resolução nº 31/123, de 1973, instituiu o ano de 1981 como o Ano Internacional das Pessoas com Deficiência (MAZZOTA, 1996). Assim, a fim de garantir a efetivação das ações propostas, foi promulgada a "Carta dos anos 80", que declarava as prioridades para o período compreendido entre os anos 80 e 90, cabendo às nações membros a preparação de um plano de ação voltado à questão da deficiência. A partir daí, o governo começa a desenvolver ações mais efetivas para essa população.

Posteriormente, foram realizados diversos encontros e fóruns, com o intuito de formar uma consciência nacional, capaz de influir na Política Nacional de Educação Física, voltada à Educação Especial. Entre esses encontros, destacam-se o I Fórum Nacional "O Excepcional e a Política de Educação Física, Desportos e Esporte para Todos”, realizado em janeiro de 85, no Rio de Janeiro; o Encontro Nacional "A pessoa deficiente e sua problemática", em junho de 85 em Brasília; o "Encontro de Professores de Educação Física", realizado em Tramandaí/RS, e o "Encontro de Avaliação do Sub-Programa de Recursos Humanos para a Educação Especial”, realizado em Batatais/SP, estes dois últimos realizados em 1986 e patrocinados pela SEED. Os participantes do encontro de Batatais, por sua vez, elaboraram a "Carta de Batatais", em que é enfatizada a importância da EF para as pessoas com deficiência, assim como a omissão dos órgãos públicos na formação de profissionais capacitados para atuar na área.

No de 1987, em meio às reformulações teóricas, debates e discussões sobre a formação e atuação do professor de EF instituiu-se as Diretrizes Curriculares Nacionais (BRASIL, 1987), com a exigência de que os cursos de formação inicial de professores ofereçam uma disciplina que trate do conhecimento sobre pessoas com deficiência, como resposta às discussões organizadas pela SEED/MEC, e reivindicações incluídas na Carta de Batatais (RIBEIRO e ARAÚJO, 2004).

\footnotetext{
${ }^{4}$ Estiveram presentes, neste encontro, uma comissão de especialistas atuantes na área, representantes da SEED, CENESP, COROE, INEP (Instituto Nacional de Estudos e Pesquisas Educacionais), CAPES (Coordenadoria de Aperfeiçoamento de Pessoal de Nível Superior) e CNPq (Conselho Nacional de Desenvolvimento Científico e Tecnológico) (LIMA, 1998).
} 
Outros avanços ocorreriam nos anos 90, com a criação do Plano Plurianual para os anos 1991/1995; a elaboração da Política Nacional de Educação Especial, sob a coordenação da SEESP/MEC, e da Proposta de Inclusão de Itens ou Disciplinas acerca de Portadores de Necessidades Especiais nos Currículos dos Cursos de $2^{\circ}$ e $3^{\circ}$ graus, ambas em 1993. Especificamente em relação ao ensino superior, a referida proposta previa a inclusão da disciplina "Aspectos ético-político-educacionais da normalização e integração da pessoa portadora de necessidades especiais", nos cursos de Pedagogia e Psicologia e em todas as licenciaturas em nível de $3^{\circ}$ grau, e conteúdos para os cursos do grupo de Ciências da Saúde, no curso de Serviço Social e nos demais cursos superiores.

Por último, convém destacar alguns pontos relevantes que aparecem nas diretrizes curriculares nacionais para o curso de graduação em EF e que discutem temas relacionados à formação profissional e as pessoas com deficiência.

O Parecer CNE/CES nº. 58/04 (BRASIL, 2004) demonstra algumas preocupações em relação às pessoas com deficiência agrupadas em quatro itens: perfil acadêmicoprofissional do graduado em EF, competências e habilidades desse profissional, estrutura e organizações curriculares dos cursos de graduação em EF e mecanismos de indissolubilidade entre teoria e prática.

$\mathrm{O}$ perfil acadêmico-profissional do graduado em EF é apresentado como um profissional que deve ser o mediador entre seres humanos e propiciar a prática dessa disciplina independentemente de idade, condições socioeconômicas, físicas e mentais, gênero, etnia e crença. Para que isso ocorra, ele deve estar qualificado para analisar criticamente a realidade social. Dentre as competências e habilidades, salienta-se a importância de saber transformar o conhecimento adquirido nas disciplinas presentes na matriz curricular do curso, além de:

Diagnosticar os interesses, as expectativas e as necessidades das pessoas (crianças, jovens, adultos, idosos, pessoas portadoras de deficiências, de grupos e comunidades especiais) de modo a planejar, prescrever, ensinar, orientar, assessorar, supervisionar, controlar e avaliar projetos e programas de atividades físicas, recreativas e esportivas nas perspectivas da prevenção, da promoção, da proteção e da reabilitação da saúde, da formação cultural, da educação e da reeducação motora, do rendimento físico-esportivo, do lazer e de outros campos que oportunizem ou venham a oportunizar a prática de atividades físicas, recreativas e esportivas (BRASIL, 2004, p. 2). 
As estruturas e organizações curriculares dos cursos de graduação em EF também são abordadas nas diretrizes curriculares, confiando às IES a responsabilidade de organizar matriz curricular de acordo com a realidade social (BRASIL, 2004, p. 3), sendo que no parágrafo 4 aponta que "as questões pertinentes às peculiaridades regionais, às identidades culturais, à educação ambiental, ao trabalho, às necessidades das pessoas portadoras de deficiência e de grupos e comunidades especiais deverão ser abordadas no trato dos conhecimentos da formação do graduado em Educação Física” (BRASIL, 2004, p. 3).

Vemos, assim, que essas diretrizes curriculares de formação, subsidiados pelo parecer 0058/2004, apontam caminhos e também apresentam elementos que reafirmam o lugar e o estudo das pessoas com deficiência nos cursos de formação em EF.

\section{A EFA NOS CURRÍCULOS DOS CURSOS DE EDUCAÇÃO FÍSICA}

Alguns estudos têm avaliado como a EFA tem contribuído para a formação de professores no Brasil. Esses estudos trazem informações sobre como esse conhecimento vem sendo tratado nos currículos dos cursos de formação de professores de EF.

Cidade, Freitas e Pedrinelli (2001) construíram um mapeamento da situação da área de EFA nos cursos de formação de professores de EF no Brasil, licenciatura e bacharelado, destacando que, das 38 instituições que responderam ao questionário, todas ofereciam uma disciplina que tratava da especificidade das pessoas com deficiência, sendo que apenas em uma delas a disciplina era ofertada como optativa. A pesquisa identificou, ainda, uma diversidade entre as nomenclaturas utilizadas.

A imprecisão conceitual em relação ao termo EFA e à população atendida por ela também foi relatada no estudo de Gonçalves (2002), que investigou a implantação e o desenvolvimento da disciplina EFA nas IES no Estado de Goiás. Foram analisados oito programas de ensino e realizadas entrevistas a seis professores de seis instituições do Estado. Os resultados indicaram variações nos aspectos pertinentes à carga horária, número de disciplinas que abordam a EFA em cada instituição, assim como a forma de distribuição organizada em períodos e séries. A autora constatou que a essência da formação inicial em 


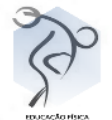

EFA no Estado situa-se no Conhecimento Técnico-funcional Aplicado, reforçando o conhecimento biológico, funcional e de desenvolvimento da deficiência. Além disso, nos programas de ensino, as atividades de pesquisa e de extensão, e o uso de novas tecnologias não são apontados.

Um estudo realizado por Lima (2005) também relata a diversidade de nomenclaturas existentes nos cursos de EF para fazer referência à disciplina EFA. De um total de 119 cursos identificados, 105 deles constavam componentes curriculares relacionados à EFA (nomenclatura mais utilizada), sendo que em apenas 14 cursos, não havia referência a um componente curricular específico no currículo para tratar do conhecimento relacionado a essa área.

O estudo de Silva (2005) buscou identificar quem eram os professores de EFA em sete IES do Estado de Mato Grosso do Sul, bem como as referências tomadas por esses para tratar o tema na formação de profissionais e professores de EF, através de questionários para docentes da disciplina EFA, e análise dos ementários e planos de ensino. Os resultados indicaram que a maioria dos professores não teve, em sua formação inicial, nenhuma disciplina que abordasse conteúdos em relação às pessoas com deficiência, buscando nos cursos de pós-graduação elementos compensadores. Por outro lado, uma parte das bibliografias indicadas pelos docentes para o planejamento da disciplina EFA não condiziam com a especificidade da área.

Gomes (2007) apresenta um panorama da disciplina EFA em oito IES públicas do Estado do Paraná. Participaram do estudo onze professores da disciplina e seus respectivos alunos, que totalizaram 463. Para coleta de dados, utilizou um questionário para os acadêmicos e uma entrevista semiestruturada para os professores. O autor destaca a prevalência de uma base pedagógica médica em detrimento da educacional na organização e trato com o conhecimento pelos professores e a aceitação deles quanto à inclusão dos alunos com deficiência nas aulas de EF. Já os alunos manifestam ser essa uma disciplina importante na formação, no entanto, destacaram existir lacunas em sua organização que limitam a preparação para lidar com esse público em situação de inclusão escolar.

Também no Estado do Paraná, Molari (2011) buscou identificar, através de um questionário que foi aplicado aos professores da disciplina EFA em 12 universidades e análise 


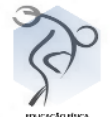

dos programas de ensino, se o paradigma da inclusão está presente nos cursos de licenciatura em EF. Os resultados indicaram que a quantidade de horas destinadas ao tema inclusão é insuficiente, e que muitos docentes apresentam inúmeras dúvidas sobre como abordar essas questões, ou até mesmo não sabem o que desenvolver para obterem um ambiente inclusivo. Por fim, observou que o ensino se perde em questões relacionadas à adaptação de atividades e em contemplações acerca de aspectos biológicos.

Com o objetivo de identificar e analisar as características das disciplinas que compõem a área da EFA nas matrizes curriculares dos cursos de EF no Brasil, Borella (2010) analisou os dados oriundos de um questionário respondido pelos coordenadores dos cursos de 114 IES, sendo 101 cursos de licenciatura e 59 cursos de bacharelado. Os resultados apontaram um alto índice de diferentes nomenclaturas e carga horária variada para as disciplinas relacionadas à EFA, assim como uma proporção pequena de docentes que apresentam titulações apropriadas à docência na área da EFA. Quanto ao Estágio Supervisionado, o estudo demonstrou que poucos cursos (28,75\%) oferecem Estágio Supervisionado na área. Por outro lado, apenas 25 cursos não proporcionam práticas como componentes curriculares. Outros 135 oferecem.

Oliveira (2011), em seu estudo, objetivou conhecer, refletir e tecer críticas ao conhecimento produzido na área de Educação Física Especial, na perspectiva inclusiva, e ao modo como esse vem sendo abordado nos currículos de formação de professores de EF na Bahia. Foi realizada também a análise dos planos de ensino de 11 cursos de Licenciatura em EF e entrevistas com sete professores. A pesquisa identificou que o trato com o conhecimento enfatiza, como desafio, formar professores para atuar numa perspectiva inclusiva, mas a análise dos planos de ensino indica a predominância de uma prática tradicional, técnica e "biologizante" que reduz muitas vezes o escopo do trabalho com pessoas com deficiência. Por outro lado, na fala dos professores entrevistados, o principal objetivo da área é o de sensibilizar os alunos em formação para o trabalho com a Educação Física Especial e a interação com pessoas com deficiência, quando deveria prever também aos estudantes em formação o domínio de conhecimento sobre a realidade e o processo de inclusão.

O estudo de Silva e Drigo (2012) teve como objetivo averiguar como a disciplina curricular EFA tem se apresentado nos cursos de licenciatura e graduação/bacharelado em 


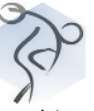

Educação Física e Esporte nas IES do Estado de São Paulo. Foram analisados 20 conteúdos programáticos (10 de IES privadas e 10 de IES públicas), sendo constatada uma diversidade de nomenclatura para a disciplina EFA. Também foram realizadas entrevistas com seis professores da disciplina de três IES públicas. Os resultados indicam que os conteúdos trabalhados têm como base o modelo médico da deficiência. Em relação às estratégias de ensino, constatou-se uma preocupação com a relação teoria e prática no ensino da disciplina, com destaque para o contato com pessoas com deficiências nas aulas no ambiente universitário e a importância dada às atividades de extensão universitária como suporte para o ensino da disciplina. $\mathrm{O}$ estudo também revela um consenso entre os participantes de que não há utilização dos conteúdos da disciplina EFA em outras disciplinas, e que está isolada no currículo.

No Rio de Janeiro, Moura et al. (2014) investigaram a formação em EFA em três IES particulares do município dos novos profissionais que poderão intervir no esporte adaptado. Os resultados encontrados apontam que os professores da disciplina EFA possuem experiência e formação profissional no esporte adaptado, entretanto, faltam aulas com atividades práticas de intervenção, e não existem atividades de extensão e parcerias das IES com qualquer instituição ligada às pessoas com deficiência. A análise das bibliografias utilizadas revela que estas atendem as novas demandas da área.

Dessa forma, a influência dos paradigmas tradicionais da área (o paradigma esportivo e o modelo médico da deficiência) ainda hoje intervém na organização desse campo de saber e no seu tratamento na formação acadêmica, fazendo com que prevaleça na maioria das vezes um olhar biomédico em detrimento do estudo de aspectos socioculturais, pedagógicos e sócio corporais da área em questão.

\section{OS CONTEÚDOS ABORDADOS NAS DISCIPLINAS DE EFA NOS CURRÍCULOS DOS CURSOS DE EDUCAÇÃO FÍSICA}

Assim como a inserção da disciplina EFA nos currículos de Educação Física, tanto no âmbito da Licenciatura, quanto no âmbito do Bacharelado, os conteúdos abordados pelas respectivas disciplinas seguem na mesma direção, ainda em estruturação. Ressalta-se 


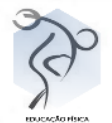

que, semelhante à fundamentação originária nas ciências biológicas da área da Educação Física, o segmento de estudos da Adaptada também tem a mesma fundamentação.

$\mathrm{Na}$ perspectiva dos cursos de Bacharelado, as disciplinas seguem, em grande parte, o padrão "biologizante" dos conteúdos, nas quais são estudadas as características morfofisiológicas das deficiências, as respostas às intervenções e suas possibilidades de ação.

Além da denominação de EFA, a disciplina pode ser apresentada, por exemplo, como Esporte Adaptado, ou esta existir como mais uma disciplina no curso, em poucos casos. Com isso pode-se perceber um teor de esportivização na fundamentação dos conteúdos, em especial ilustrados pelas Paralimpíadas, que a cada edição vem se tornando mais destaque na sociedade. Nota-se nessa área, inclusive, um maior avanço com relação às pesquisas e produções científicas, principalmente no âmbito de pós graduação stricto-senso (SILVA e ARAÚJO, 2012), gerando conhecimento científico em diversas modalidades (ARGENTIN et al., 2013; SILVA et al. 2013; PASINI et al., 2014).

Dentre as modalidades institucionalizadas pelo Comitê Paralímpico Brasileiro estão: atletismo, basquete em cadeira de rodas, bocha, ciclismo, esgrima em cadeira de rodas, futebol de 5, futebol de 7, goalball, halterofilismo, hipismo, judô, natação, paracanoagem, remo, rugby, tênis de mesa, tênis em cadeira de rodas, tiro com arco, tiro esportivo, triatlo, vela, voleibol sentado.

Sendo assim, os conteúdos abordados no Bacharelado perpassam pelos ajustes e adaptações das atividades físicas/exercícios físicos/treinamentos em pessoas com deficiência, relação saúde-doença, o esporte específico (como, por exemplo, o goalball, modalidade desenvolvida exclusivamente para as pessoas com deficiência) ou o adaptado (como o basquete em cadeira de rodas, ou voleibol sentado, que são as modalidades institucionalizadas de forma adaptada).

Já nas Licenciaturas, devido às mudanças de paradigmas, pela característica e configuração do curso, a disciplina relacionada à EFA vem se caracterizando fortemente pela discussão política-histórica-social da pessoa com deficiência e sua inclusão, tanto na sociedade, quanto, mais especificamente, nos conteúdos da Educação Física Escolar, inclusive embasada nas Diretrizes para a Educação Especial na Educação Básica, Resolução CNE/CEB n. 2/2001 (BRASIL, 2001). Nesse âmbito as disciplinas podem ser apresentadas, por 


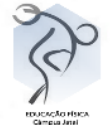

exemplo, com as denominações Educação Física Especial e Educação Física Escolar Adaptada, Metodologia de Ensino em EFA, entre outras.

Nesse cenário, em geral, o planejamento dos conteúdos vão na direção das discussões acerca da inserção da pessoa com deficiência na sociedade e seus direitos, as políticas públicas que se relacionam com essa população, reflexões sobre como desenvolver os conteúdos específicos da área, normalmente os conteúdos da cultura corporal (jogos, dança, lutas, esporte e ginástica) (CASTELLANI FILHO et al., 2009) em um panorama inclusivo, onde as atividades sejam adaptadas, permitindo a participação de todos.

Com isso, observa-se a preocupação da participação ampla, mas pouco se desenvolve acerca de atividades específicas. É como se a pessoa com deficiência tivesse o direito à participação uma vez que a atividade foi adaptada (RODRIGUES, 1991). No entanto, uma atividade que originalmente seja desenvolvida para qualquer participação, que já preveja as diferenças, e que todos os alunos desenvolvam todo o seu potencial, (LIEBERMAN, HOUSTON-WILSON, KOZUB, 2002; O'BRIEN, KUDLACEK, HOWE, 2009) normalmente são restritas.

Por fim, faz-se necessária uma maior sistematização dos conteúdos a serem abordados durante a oferta das disciplinas relacionadas à EFA nos cursos de graduação para que a atuação se aproxime dos desafios e realidades da sociedade.

\section{CONSIDERAÇÕES FINAIS}

Após as reflexões apresentadas é possível perceber o avanço da abordagem e do trato dos conteúdos relacionados à pessoa com deficiência nos currículos de formação em Educação Física. A área de EFA, tanto no ensino de graduação, quanto na pós-graduação tem seu roteiro ainda sob escrita, com muito a ser aperfeiçoado. Observa-se que tem sido delegado ao professor da disciplina específica, no caso a EFA, fazer toda a adaptação dos conhecimentos necessários à formação, ou seja, apenas a EFA tem a obrigação de pensar o deficiente. 
No entanto, a sociedade também deve ser adaptada (do ponto de vista estrutural, físico, conceitual e atitudinal) para fazer parte efetiva da inclusão social, já que um dos pontos de maior entrave para o desenvolvimento do deficiente está na impossibilidade ter uma educação que auxilia a superar problemas na socialização, que estimule o aprendizado, e contribua para a qualificação profissional da pessoa com deficiência.

A formação na área com conhecimento técnico e científico se faz ordinária, uma vez que a relação da pessoa com deficiência na sociedade vem ganhando espaço com direitos e políticas públicas cada vez mais presentes. O lidar com o diferente em uma classe inclusiva é extremamente complicado quando não se têm os instrumentos metodológicos e psicossociais bem trabalhados. Os cursos de formação em Educação Física, sobretudo as licenciaturas ainda trabalham em uma perspectiva voltada para um aluno "padrão", e uma prática predominantemente tradicional e técnica. Nesse contexto, os grupos considerados minorias, nos quais se encontram os alunos com deficiência, não são levados em consideração pela maior parte do professorado, mesmo sabendo que o universo dos alunos nas salas de aula das escolas (principalmente as públicas) é uma realidade bem diferente.

Buscar uma formação que possa às futuras demandas sociais/escolares inclusivas implica uma reavaliação dos fatores que influenciam na formação. $\mathrm{O}$ currículo tem que ser modificado em todos os âmbitos, de forma que as discussões e políticas públicas bem intencionadas não se esbarrem na praticidade da realidade concreta. Nesse sentido, não basta apenas ter a disciplina EFA no currículo e sem contextualização no tripé ensino/pesquisa/extensão e sim proporcionar as ferramentas para que os futuros educadores sejam capazes de posicionar-se de forma competente diante da diferença.

\section{REFERÊNCIAS}

ARAÚJO, Paulo Ferreira de. Desporto adaptado no Brasil: origem, institucionalização e atualidade. Brasília: MEC/INDESP, 1998. 


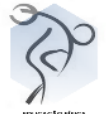

ARGENTIN, Rodolfo et al. Quantificação da distância percorrida, velocidade média e máxima em um jogo de rugby em cadeira de rodas: estudo piloto. Caderno de Educação Física e Esporte, v. 11, p. 57-63, 2013.

BETTI, Irene C. Rangel; BETTI, Mauro. Novas perspectivas em formação profissional. Motriz, Rio Claro, v.2, n.1, p.10-15, 1996.

BORELLA, Douglas Roberto. Atividade física adaptada no contexto das matrizes curriculares dos cursos de educação física. São Carlos: UFSC, 2010. Tese (Doutorado em Educação Especial) - Centro de Educação e Ciências Humanas, Universidade Federal de São Carlos, 2010.

BRASIL. Conselho Federal de Educação. Resolução n. 3, 16 de janeiro de 1987. Diário Oficial. (172). Brasília, set. 1987.

Diretrizes Nacionais para a Educação Especial na Educação Básica. Brasília: MEC/SEESP, 2001.

Ministério da Educação. Conselho Nacional de Educação. Câmara de Ensino Superior. Parecer n. 0058, de 18 de fevereiro de 2004. Diretrizes curriculares nacionais para os cursos de graduação em educação física. Brasília, 2004.

CARMO, Apolônio Abadio do. Deficiência física: a sociedade brasileira cria, recupera e discrimina. Brasília: Secretaria dos Desportos, 1991.

CASTELlANi FILHO, Lino et al. Metodologia do Ensino de Educação Física. 2 ed ver. São Paulo: Editora Cortez, 2009.

CIDADE, Ruth Eugênia; FREITAS, Patrícia S.; PEDRINELLI, Verena J. In: Revista da SOBAMA: Temas em Educação Física Adaptada, 2001, p. 86-91.

COSTA, Alberto Martins. Formação de recursos humanos para educação física adaptada. In: IV Simpósio Paulista de Educação Física Adaptada, 1992, São Paulo, Anais... São Paulo, 1992.

CPB. Comitê Paralímpico Brasileiro. Modalidades esportivas. 2016. Disponível em: http://www.cpb.org.br/.

DUARTE, Edison. A formação do profissional em atividade motora adaptada. In: MENDES, Enicéia Gonçalves; ALMEIDA, Maria Amélia; WILLIAMS, Lúcia. C. A. (Orgs.). Temas em Educação Especial: avanços recentes. São Carlos, SP: EdUFSCAR, 2004, p. 35 a 56.

GOMES, Nilton Munhoz. Análise da disciplina educação física especial nas instituições de ensino superior públicas do estado do Paraná. Campinas: UNICAMP, 2007. Tese 


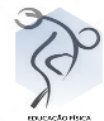

(Doutorado em Educação Física) - Faculdade de Educação Física, Universidade Estadual de Campinas, 2007.

GONÇALVES, Vivianne Oliveira; Estudo da disciplina educação física adaptada nas instituições de ensino superior no estado de Goiás. Campinas: UNICAMP, 2002. Dissertação (Mestrado em Educação Física) - Faculdade de Educação Física, Universidade Estadual de Campinas, 2002.

LIEBERMAN, Lauren J.; HOUSTON-WILSON, Cathy; KOZUB, Francis M. Perceived barriers to including students with visual impairments in general physical education. Adapted Physical Activity Quartely, Illinois, v. 19, p. 364-377, 2002.

LIMA, Solange Rodovalho. Cursos de especialização em educação física e esportes adaptados: onde estão seus egressos? Uberlândia: UFU, 1998. Dissertação (Mestrado em Educação) - Faculdade de Educação, Universidade Federal de Uberlândia, 1998.

LIMA, Sônia Maria T. Educação física adaptada: proposta de ação metodológica para formação universitária. Campinas: UNICAMP, 2005. Tese (Doutorado em Educação Física) Faculdade de Educação Física, Universidade Estadual de Campinas, 2005.

MAZZOTA, Marcos J. S. Educação especial no Brasil: história e políticas públicas. São Paulo: Cortez, 1996.

MOLARI, Mário. Inclusão escolar e formação profissional em educação física: o caso das universidades no Estado do Paraná. Ribeirão Preto: CUML, 2011. Dissertação (Mestrado em Educação) - Centro Universitário Moura Lacerda, 2011.

MOURA, Diego Luz et al. A disciplina educação física adaptada nos cursos de graduação no Rio de Janeiro. Conexões, v. 12, n. 14, p. 1-12, 2014. Disponível em: < http://fefnet178.fef.unicamp.br/ojs/index.php/fef/article/view/858/pdf>. Acesso em 10 de janeiro de 2015.

O'BRIEN, Deirdre; KUDLACEK, Martin; HOWE, Peter David. A contemporary review of English language literature on inclusion of students with disabilities in physical education: a European perspective. European Journal of Adapted Physical Activity, Olomuc, v. 2, n. 1, p. 46-61, 2009.

OLIVEIRA, João Danilo B. Educação física especial e currículo: (in) formação para a educação inclusiva. Salvador: UFBA, 2011. Tese (Doutorado em Educação) - Faculdade de Educação, Universidade Federal da Bahia, 2011.

PASINI, Gustavo Moreira et al. Processo de institucionalização do esporte paralímpico brasileiro: caracterização do caso da equipe de rugby em cadeiras rodas da Unicamp/Adeacamp. Conexões Campinas, v. 12, p. 82-93, 2014. 


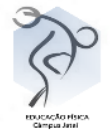

RIBEIRO, Sônia Maria; ARAÚJO, Paulo Ferreira de. A formação acadêmica refletindo na expansão do desporto adaptado: uma abordagem brasileira. Revista Brasileira de Ciências do Esporte, v. 25, n. 3, p. 7-160, maio 2004.

RODRIGUES, Júlio L. Educação física no contexto interdisciplinar e a pessoa portadora de deficiência. Piracicaba: UNIMEP,1991. Dissertação (Mestrado em Educação Física) Faculdade de Educação Física, Universidade Metodista de Piracicaba, 1991.

SILVA, Cláudio Silvério da; DRIGO, Alexandre Janotta. A educação física adaptada: implicações curriculares e formação profissional. São Paulo: Cultura Acadêmica, 2012.

SILVA, Marília Magno et al. Sport injuries in elite paralympic swimmers with visual impairment. Journal of Athletic Training, v. 48, p. $130614075738001-000,2013$.

SILVA, Rita de Fátima da. A ação do professor de ensino superior na educação física adaptada. Campinas: UNICAMP, 2005. Dissertação (Mestrado em Educação Física) Faculdade de Educação Física, Universidade Estadual de Campinas, 2005.

SILVA, Rita de Fátima da.; ARAÚJO, Paulo Ferreira de. A educação física adaptada e o percurso para sua alocação enquanto disciplina na formação superior. Conexões, Campinas, v. 3, n. 2, 2005. 